subject of investigations by different authors with different methods and in various contexts (cf. [2]). The concept of a totally regular variable, introduced by $\mathrm{R}$. Delanghe in [5] and later also studied by Gürlebeck ([7], [9]) for the special case of quaternions, has some obvious relationship with the latter. It describes the set of linear monogenic functions whose integer powers are also monogenic (without demanding to form an Appell sequence as it is the case for the integer powers of the complex variable $z=x+i y)$. Indeed, the simple example of the totally regular Fueter-polynomials (cf. [10], [12]) shows, that not every totally regular variable and its integer powers form an Appell sequence with respect to the hypercomplex derivative. From the other side, the Appell sequence constructed in [6] is not constituted by a totally regular variable and its integer powers.

These facts motivated us to ask for the relationship between totally regular variables and Appell sequences with respect to the hypercomplex derivative in the case of a paravector valued variable in $\mathbb{R}^{3}$. Therefore we characterize completely the set of all paravector valued totally regular variables. The higher dimensional case can be treated in the same way. In view of our aim to connect totally regular variables with Appell sequences, we are using a natural normalization condition for the set of all paravector valued totally regular variables. We prove that under that normalization condition all totally regular variable constitute automatically Appell sequences, since they are isomorphic to the complex variables. We finish with some remarks on the role of polynomials in terms of the totally regular Fueter-polynomials (which are not normalized in the aforementioned way) as well as their use in the construction of Appell sequences with respect to the hypercomplex derivative.

\section{Basic Notations}

As usual, let $\left\{e_{1}, e_{2}, \ldots, e_{n}\right\}$ be an orthonormal basis of the Euclidean vector space $\mathbb{R}^{n}$ with a non-commutative product according to the multiplication rules

$$
e_{k} e_{l}+e_{l} e_{k}=-2 \delta_{k l}, \quad k, l=1, \ldots, n,
$$

where $\delta_{k l}$ is the Kronecker symbol. The set $\left\{e_{A}: A \subseteq\{1, \ldots, n\}\right\}$ with

$$
e_{A}=e_{h_{1}} e_{h_{2}} \cdots e_{h_{r}}, \quad 1 \leq h_{1}<\cdots<h_{r} \leq n, \quad e_{\emptyset}=e_{0}=1,
$$

forms a basis of the $2^{n}$-dimensional Clifford algebra $C \ell_{0, n}$ over $\mathbb{R}$. Let $\mathbb{R}^{n+1}$ be embedded in $C \ell_{0, n}$ by identifying $\left(x_{0}, x_{1}, \ldots, x_{n}\right) \in \mathbb{R}^{n+1}$ with

$$
x=x_{0}+\underline{x} \in \mathcal{A}_{n}:=\operatorname{span}_{\mathbb{R}}\left\{1, e_{1}, \ldots, e_{n}\right\} \subset C \ell_{0, n} .
$$

Here, $x_{0}=\operatorname{Sc}(x)$ and $\underline{x}=\operatorname{Vec}(x)=e_{1} x_{1}+\cdots+e_{n} x_{n}$ are, the so-called, scalar and vector parts of the paravector $x \in \mathcal{A}_{n}$. The conjugate of $x$ is given by $\bar{x}=x_{0}-\underline{x}$ and its norm by $|x|=(x \bar{x})^{\frac{1}{2}}=\left(x_{0}^{2}+x_{1}^{2}+\cdots+x_{n}^{2}\right)^{\frac{1}{2}}$.

To call attention to its relation to the complex Wirtinger derivatives, we use the following notation for a generalized Cauchy-Riemann operator in $\mathbb{R}^{n+1}, n \geq$ 1 :

$$
\bar{\partial}:=\frac{1}{2}\left(\partial_{0}+\partial_{\underline{x}}\right), \quad \partial_{0}:=\frac{\partial}{\partial x_{0}}, \quad \partial_{\underline{x}}:=e_{1} \frac{\partial}{\partial x_{1}}+\cdots+e_{n} \frac{\partial}{\partial x_{n}} .
$$


Definition 1 (Monogenic function).

$\mathcal{C}^{1}$-functions $f$ satisfying the equation $\bar{\partial} f=0$ (resp. $\left.f \bar{\partial}=0\right)$ are called left monogenic (resp. right monogenic).

We suppose that $f$ is hypercomplex-differentiable in $\Omega$ in the sense of $[8,12]$, that is, it has a uniquely defined areolar derivative $f^{\prime}$ in each point of $\Omega$ (see also [13]). Then, $f$ is real-differentiable and $f^{\prime}$ can be expressed by real partial derivatives as $f^{\prime}=\partial f$ where, analogously to the generalized Cauchy-Riemann operator, we use $\partial:=\frac{1}{2}\left(\partial_{0}-\partial_{\underline{x}}\right)$ for the conjugate Cauchy-Riemann operator. Since a hypercomplex differentiable function belongs to the kernel of $\bar{\partial}$, it follows that, in fact, $f^{\prime}=\partial_{0} f=-\partial_{x} f$ which is similar to the complex case.

In general, $C \ell_{0, n}$-valued functions defined in some open subset $\Omega \subset \mathbb{R}^{n+1}$ are of the form $f(z)=\sum_{A} f_{A}(z) e_{A}$ with real valued $f_{A}(z)$. However, in several applied problems it is very useful to construct $\mathcal{A}_{n}$-valued monogenic functions as functions of a paravector with special properties. In this case we have

$$
f\left(x_{0}, \underline{x}\right)=\sum_{j=0}^{n} f_{j}\left(x_{0}, \underline{x}\right) e_{j}
$$

and left monogenic functions are also right monogenic functions, a fact which follows easily by direct inspection of the corresponding real system of first order partial differential equations (generalized Riesz system).

\section{Example 1.}

1. Consider the $\mathcal{A}_{3}$-valued function

$$
f(x)=f\left(x_{0}, x_{1}, x_{2}, x_{3}\right)=x_{1} x_{2} x_{3}-x_{0} x_{2} x_{3} e_{1}-x_{0} x_{1} x_{3} e_{2}-x_{0} x_{1} x_{2} e_{3} .
$$

Simple calculations allow to conclude that $\bar{\partial} f=0$ which means that $f$ is left monogenic. Since $f$ is of the form (1), it follows that $f$ is also right monogenic. Moreover, $f^{\prime}(x)=\partial_{0} f(x)=-x_{2} x_{3} e_{1}-x_{1} x_{3} e_{2}-x_{1} x_{2} e_{3}$.

2. Consider now the $\mathcal{A}_{2}$-valued functions

$$
f_{k}\left(x_{0}, x_{1}, x_{2}\right)=\left(x_{0}+x_{1} e_{1}+x_{2} e_{2}\right)^{k}, k=1,2, \ldots
$$

It follows easily that

$$
\begin{aligned}
& \bar{\partial} f_{1}=-1 \\
& \bar{\partial} f_{2}=-2 x_{0} \\
& \bar{\partial} f_{3}=-3 x_{0}^{2}+\left(x_{1}^{2}+x_{2}^{2}\right) ; \\
& \bar{\partial} f_{4}=\left(-4 x_{0}^{2}+4\left(x_{1}^{2}+x_{2}^{2}\right)\right) x_{0} .
\end{aligned}
$$

In fact, by induction, on can prove that

$$
\bar{\partial} f_{n}=\left\{\begin{array}{l}
\sum_{k=0}^{r-1}(-1)^{1+k}\left(\begin{array}{c}
2 r \\
2 k+1
\end{array}\right) x_{0}^{2 r-1-2 k}\left(x_{1}^{2}+x_{2}^{2}\right)^{k}, \text { if } n=2 r \\
\sum_{k=0}^{r}(-1)^{1+k}\left(\begin{array}{c}
2 r+1 \\
2 k+1
\end{array}\right) x_{0}^{2 r-2 k}\left(x_{1}^{2}+x_{2}^{2}\right)^{k}, \text { if } n=2 r+1 .
\end{array}\right.
$$


Therefore, neither $z:=f_{1}(x)$ nor any of its nonnegative integer powers are left or right monogenic functions.

We use also the classical definition of sequences of Appell polynomials [1] adapted to the hypercomplex case.

Definition 2 (Generalized Appell sequence).

A sequence of monogenic polynomials $\left(\mathcal{P}_{k}\right)_{k \geq 0}$ of exact degree $k$ is called a generalized Appell sequence with respect to the hypercomplex derivative if

1. $\mathcal{P}_{0}(x) \equiv 1$,

2. $\mathcal{P}_{k}^{\prime}=k \mathcal{P}_{k-1}, k=1,2, \ldots$

The second condition is the essential one while the first condition is the usually applied normalization condition which can be changed to any constant different from zero.

\section{Totally Regular Variables}

Underlining the fact that, in general, an integer power of a hypercomplex variable is not monogenic, Delanghe introduced the following concept (see [5])

Definition 3 (Totally regular variable).

A totally regular variable is a linear monogenic function of the form

$$
z=x_{0} e_{A_{0}}+x_{1} e_{A_{1}}+\ldots+x_{n} e_{A_{n}} \in C \ell_{0, n}
$$

whose integer powers are monogenic.

Depending on the choice of $e_{A_{k}}$, Delanghe obtained for the general Clifford Algebra valued case, where $e_{i}^{2}=\varepsilon_{i} e_{0}$, for real $\varepsilon_{i}, \quad(i=1, \ldots, n)$, necessary and sufficient conditions for a hypercomplex variable $z$ to be totally regular $[5$, Theorem 4]. For our purpose here, we would like to call attention to the following weaker result, involving a much simpler condition.

Theorem 1. [5, Corollary 1 of Theorem 4] Any monogenic variable $z$ of the form (2) for which

$$
e_{A_{k}} e_{A_{l}}=e_{A_{l}} e_{A_{k}}, \quad k, l=0, \ldots, n,
$$

is totally regular.

Additionally, Delanghe showed that (3) is only sufficient, by referring to the special case of the totally regular variable $z=x_{2} e_{1} e_{2}+x_{3} e_{1} e_{3}$, with $e_{1}^{2}=\varepsilon_{1} \neq$ $0, e_{2}^{2}=e_{3}^{2}=0$, for which clearly $e_{1} e_{2} \cdot e_{1} e_{3} \neq e_{1} e_{3} \cdot e_{1} e_{2}$.

Later on Gürlebeck [7] studied the case of quaternion valued ( $\mathbb{H}$ - valued) variables in the form of

$$
z=x_{0} d_{0}+x_{1} d_{1}+x_{2} d_{2}+x_{3} d_{3} \in \mathbb{H},
$$


with $d_{k}=a_{k 0} e_{0}+a_{k 1} e_{1}+a_{k 2} e_{2}+a_{k 3} e_{1} e_{2}$ not necessarily linearly independent (see also [9]). In order to obtain $\mathbb{H}$-totally regular variables he found a necessary and sufficient condition, expressed by the rank of the matrix

$$
A=\left(\begin{array}{lll}
a_{01} & a_{02} & a_{03} \\
a_{11} & a_{12} & a_{13} \\
a_{21} & a_{22} & a_{23} \\
a_{31} & a_{32} & a_{33}
\end{array}\right),
$$

which can be rewritten as follows:

Theorem 2. Let $z$ be a quaternionic holomorphic variable of the form (4). The following statements are equivalent:

I. $\quad z$ is a a totally regular variable;

II. $\quad d_{k} d_{l}=d_{l} d_{k}, l, k=0,1,2,3$;

III. The rank of the matrix (5) is less than 2 .

We note that the general form of a totally regular variable has not been explicitly determined, neither in the general case (2) nor in the quaternionic case (4). The aim of the present work is to characterize totally regular variables defined in $\mathbb{R}^{3}$.

Following this idea we study here the case of linear paravector valued functions of three real variables, subject to a normalization condition with respect to the real variable $x_{0}$. This normalization condition is given in terms of the hypercomplex derivative by demanding that

$$
\partial z=z \partial=1
$$

This is motivated by the fact that at the same time we are looking for the characterization of all totally regular variables whose integer powers form an Appell sequence in the sense of Definition 2 as we know it from the complex case for $z=x+i y$.

We note that not every totally regular variable (TRV) and its powers form an Appell sequence. In addition the first degree polynomial of an Appell sequence is not necessarily a TRV. The following examples illustrate these situations.

Example 2.

1. The variables

$$
z_{s}:=x_{s}-x_{0} e_{s}, s=1,2,
$$

are TRV, which are not Appell sequences in the sense of Definition 2, because

$$
\bar{\partial} z_{s}^{k}=0, k=1,2, \ldots \quad \text { but } \quad \partial z_{s}=\partial_{0} z_{s}=-e_{s} \neq 1 .
$$


2. A sequence of the form considered in [6]

$$
\mathcal{P}_{k}(x)=\sum_{s=0}^{k} \frac{1}{2^{s}}\left(\begin{array}{l}
k \\
s
\end{array}\right)\left(\begin{array}{c}
s \\
\left\lfloor\frac{s}{2}\right\rfloor
\end{array}\right) x_{0}^{k-s} \underline{x}^{s},
$$

is an Appell sequence which does not consist of a TRV and its powers, since besides the fact that

$$
\tilde{z}:=\mathcal{P}_{1}(x)=x_{0}+\frac{1}{2}\left(x_{1} e_{1}+x_{2} e_{2}\right)
$$

is not a TRV, we also have $\tilde{z}^{k} \neq \mathcal{P}_{k}, k>1$.

3. The variables

$$
\hat{z}_{s}:=x_{0}+x_{s} e_{s}, s=1,2,
$$

are TRV and their powers form an Appell sequence, because

$$
\bar{\partial} \hat{z}_{s}^{k}=0 \quad \text { and } \quad \partial \hat{z}_{s}^{k}=\partial_{0} \hat{z}_{s}^{k}=k \hat{z}_{s}^{k-1}, s=1,2, \ldots
$$

\section{The Explicit Form of Paravector Valued Totally Regular Variables}

As mentioned before, for reasons of applications and simplicity we concentrate on the computation of the explicit form of TRV given by

$$
z=x_{0} d_{0}+x_{1} d_{1}+x_{2} d_{2} \in \mathcal{A}_{2} \subset C l_{0,2} \text {. }
$$

We first note that from $\bar{\partial} z=0$ it follows that

$$
d_{0}+e_{1} d_{1}+e_{2} d_{2}=0
$$

In addition, the application of the normalization condition (6) implies immediately that

$$
d_{0}=1
$$

and therefore, combining (11) and (12) we obtain as a first condition on the $d_{k}$ 's the following relation

$$
1+e_{1} d_{1}+e_{2} d_{2}=0
$$

For $z$ to be TRV we also need that the square of $z$ and all other integer powers of $z$ are monogenic. We will see, that the case of $z^{2}$ implies conditions which guarantee the same property for all integer powers. Since

$$
\begin{aligned}
\bar{\partial} z^{2}=x_{0}(1+ & \left.e_{1} d_{1}+e_{2} d_{2}\right)+\left(1+e_{1} d_{1}\right) x_{1} d_{1}++\left(1+e_{2} d_{2}\right) x_{2} d_{2} \\
& +\frac{1}{2}\left(x_{2} e_{1}+x_{1} e_{2}\right)\left(d_{1} d_{2}+d_{2} d_{1}\right) \\
=x_{0}(1+ & \left.e_{1} d_{1}+e_{2} d_{2}\right)+\left(1+e_{1} d_{1}+e_{2} d_{2}\right) x_{1} d_{1}+\left(1+e_{1} d_{1}+e_{2} d_{2}\right) x_{2} d_{2} \\
& +\frac{1}{2}\left(x_{1} e_{2}-x_{2} e_{1}\right)\left(d_{1} d_{2}-d_{2} d_{1}\right)
\end{aligned}
$$


and taking into account condition (13), we obtain a second condition on the $d_{k}$ 's, namely

$$
d_{1} d_{2}-d_{2} d_{1}=0 .
$$

Notice that (14) is identical to the necessary and sufficient conditions, mentioned in Theorem 2. For a detailed analysis of the consequences of (13) and (14) we use the notation of [7] and write

$$
\begin{aligned}
& d_{1}=a_{10}+a_{11} e_{1}+a_{12} e_{2}, \\
& d_{2}=a_{20}+a_{21} e_{1}+a_{22} e_{2},
\end{aligned}
$$

with $a_{l m} \in \mathbb{R}, l, m=0,1,2$. Therefore, from (13) it follows easily that

$$
\begin{gathered}
a_{11}+a_{22}=1, \\
a_{12}=a_{21}
\end{gathered}
$$

and

$$
a_{10}=a_{20}=0
$$

while condition (14) implies

$$
a_{11} a_{22}-a_{12} a_{21}=0 .
$$

We note that, based on (10) and (12), the matrix (5) has the form

$$
A=\left(\begin{array}{ccc}
0 & 0 & 0 \\
a_{11} & a_{12} & 0 \\
a_{21} & a_{22} & 0
\end{array}\right),
$$

which has obviously rank less than 2 , due to (18).

Relation (16) together with (18) gives

$$
a_{11} a_{22}=\lambda^{2}, \quad \text { for some real } \lambda \text {. }
$$

Let us now consider the two possible cases, for the values of the parameter $\lambda$.

Case $\mathbf{A}: \lambda \neq 0$.

In this first case, $a_{11}$ and $a_{22}$ have the same sign and as a consequence of (15), both coefficients are positive. Therefore we can define

$$
i_{1}^{2}:=a_{11} ; \quad i_{2}^{2}:=a_{22} \quad \text { with } \quad i_{1}^{2}+i_{2}^{2}=1,
$$

in order to write

$$
\lambda^{2}=\left(i_{1} i_{2}\right)^{2}
$$

Remark: Because of

$$
i_{1}^{2}+i_{2}^{2}=1
$$


we can choose, for instance,

$$
i_{1}=t, \quad i_{2}=\sqrt{1-t^{2}},\left(\text { with }|t|=\left|i_{1}\right| \leq 1\right),
$$

or

$$
i_{1}=\cos \alpha, \quad i_{2}=\sin \alpha, \text { (for some angle } \alpha \text { ). }
$$

The relation with the roots of unity is obvious and permits interesting applications (see [4]).

The consequences of case $\mathbf{A}$ for the general form of the TRV $z$ are the following:

$$
\begin{aligned}
z & =x_{0}+x_{1}\left(i_{1}^{2} e_{1}+i_{1} i_{2} e_{2}\right)+x_{2}\left(i_{1} i_{2} e_{1}+i_{2}^{2} e_{2}\right) \\
& =x_{0}+i_{1} x_{1}\left(i_{1} e_{1}+i_{2} e_{2}\right)+i_{2} x_{2}\left(i_{1} e_{1}+i_{2} e_{2}\right) \\
& =x_{0}+\left(i_{1} x_{1}+i_{2} x_{2}\right)\left(i_{1} e_{1}+i_{2} e_{2}\right),
\end{aligned}
$$

where the constant "imaginary unit"

$$
\hat{\imath}:=i_{1} e_{1}+i_{2} e_{2}
$$

is such that $\hat{\imath}^{2}=-i_{1}^{2}-i_{2}^{2}=-1$. Writing

$$
x_{\hat{\imath}}:=i_{1} x_{1}+i_{2} x_{2},
$$

we recognize the isomorphism with $z=x+y i \in \mathbb{C}$ :

$$
x \rightarrow x_{0} ; \quad y \rightarrow x_{\hat{\imath}} ; \quad i \rightarrow \hat{\imath} .
$$

Moreover, under the conditions of case A, $z$ is a TRV whose integer powers

$$
z^{k}=\left[x_{0}+\left(i_{1} x_{1}+i_{2} x_{2}\right)\left(i_{1} e_{1}+i_{2} e_{2}\right)\right]^{k}=\left(x_{0}+x_{\hat{\imath}} \hat{\imath}\right)^{k}
$$

form an Appel sequence, because obviously $\left(z^{k}\right)^{\prime}=k z^{k-1}$ and $z^{0}=1$.

Consider now the second case:

Case B: $\lambda=0$

If

$$
a_{11} \neq 0 \text { and } a_{22}=0,
$$

then $a_{11}=1$ and $z=x_{0}+x_{1} e_{1}$ (trivial case). On the other hand, if

$$
a_{11}=0 \text { and } a_{22} \neq 0 \text {, }
$$

then $a_{22}=1$ and $z=x_{0}+x_{2} e_{2}$ (also a trivial case).

The above considerations can be summarized as follows: 
Theorem 3. The set of all linear monogenic variables of the form

$$
z=x_{0}+x_{1} d_{1}+x_{2} d_{2} \in \mathcal{A}_{2} \subset C l_{0,2},
$$

which are TRV explicitly consists of pseudo-complex variables of the form

$$
z_{\hat{\imath}}=x_{0}+\left(i_{1} x_{1}+i_{2} x_{2}\right)\left(i_{1} e_{1}+i_{2} e_{2}\right)=x_{0}+x_{\hat{\imath}} \hat{\imath},
$$

with $\left(i_{1}, i_{2}\right) \in \mathbb{R}^{2}$ and $i_{1}^{2}+i_{2}^{2}=1$.

Moreover, due to their isomorphism with the complex variable $z=x+y i$ these pseudo-complex variables together with their integer powers $z_{\hat{\imath}}^{k}$ form automatically an Appell sequence with respect to the hypercomplex derivative.

\section{Concluding Remarks}

Even the consideration of homogeneous polynomials of degree $k$, with a "relaxed" binomial expansion (characteristic property of Appell sequences) of the form

$$
\mathcal{P}_{k}(z)=\sum_{0}^{k} m_{s}\left(\begin{array}{l}
k \\
s
\end{array}\right) x_{0}^{k-s}\left[X_{1}\left(x_{1}, x_{2}\right) e_{1}+X_{2}\left(x_{1}, x_{2}\right) e_{2}\right]^{s},
$$

where $X_{i}\left(x_{1}, x_{2}\right), i=1,2$, are real valued functions in $x_{1}$ and $x_{2}$, leads only to the cases $\mathbf{A}$ e $\mathbf{B}$ of TRV with $m_{s} \equiv 1$ or to the case where

$$
m_{s}=\frac{1}{2^{s}}\left(\begin{array}{c}
s \\
\left\lfloor\frac{s}{2}\right\rfloor
\end{array}\right), \quad s=0,1, \ldots, k,
$$

with $X_{1}\left(x_{1}, x_{2}\right)=x_{1}$ and $X_{2}\left(x_{1}, x_{2}\right)=x_{2}$ (not covered by $\mathbf{A}$ or $\mathbf{B}$ and not based on the integer powers of a TRV, since $\left.\mathcal{P}_{1}^{2} \neq \mathcal{P}_{2}\right)$.

Polynomials of the form (19) as elements of generalized Appell sequences of paravector valued monogenic polynomials in $\mathcal{A}_{2}$ have been studied in [14]. It was proved that both mentioned cases, i.e. where $m_{s} \equiv 1$ or $m_{s}$ given by (20), are the only one examples of Appell sequences with respect to the hypercomplex derivative and normalized as in Definition 2. This means that with the exception of polynomials (19) in the special form

$$
\mathcal{P}_{k}(z)=\sum_{0}^{k} \frac{1}{2^{s}}\left(\begin{array}{c}
s \\
\left\lfloor\frac{s}{2}\right\rfloor
\end{array}\right)\left(\begin{array}{l}
k \\
s
\end{array}\right) x_{0}^{k-s}\left(x_{1} e_{1}+x_{2} e_{2}\right)^{s},
$$

all other Appell sequences with respect to the hypercomplex derivative and normalized as in Definition 2, consist of totally regular variables (TRV) and its integer powers in the form

$$
z_{\hat{\imath}}=x_{0}+\left(i_{1} x_{1}+i_{2} x_{2}\right)\left(i_{1} e_{1}+i_{2} e_{2}\right)=x_{0}+x_{\hat{\imath}} \hat{\imath} .
$$

Further, let us mention the following. If we admit that the usually used normalization condition $\mathcal{P}_{0}=1$ (or initial value of the polynomial of degree 0 ) in 
Definition 2 is changed to $\mathcal{P}_{0}=-e_{1}$, resp. $\mathcal{P}_{0}=-e_{2}$, (a possibility that we mentioned), then also the TRV in the examples of Section 4

$$
z_{s}=x_{s}-x_{0} e_{s}=-e_{s}\left(x_{0}+x_{s} e_{s}\right), s=1,2,
$$

form together with its integer powers Appell sequences, which can be verified by straightforward calculations. The initial value appears as the constant factor $-e_{1}$ resp. $-e_{2}$ of the considered $z_{\hat{\imath}}^{k}$ with $\left(i_{1}, i_{2}\right)=(1,0)$, resp. $\left(i_{1}, i_{2}\right)=(0,1)$. Of course, the same is true for other choices of initial values of the polynomial of degree 0 and constant factors of the "natural" two copies of the complex variable $z=x+y i$, i.e. for the first degree polynomials

$$
z_{r}=x_{0}+x_{r} e_{r}, r=1,2 \text {. }
$$

But since both TRV $z_{s}$ of the form (21) are the first degree Fueter polynomials (see [10]), we mention finally a remark of Habetha in [11, p. 233], on the use of those "natural" copies of several complex variables, i.e. $x_{0}+x_{s} e_{s}=e_{s} z_{s}$, with $s=1,2$, instead of Fueter polynomials for the power series representation of any monogenic function. Theorem 3 shows (here only for the case of $\mathbb{R}^{3}$ ), that also the more general pseudo-complex variables of the form $z_{\hat{\imath}}=x_{0}+\left(i_{1} x_{1}+i_{2} x_{2}\right)\left(i_{1} e_{1}+\right.$ $\left.i_{2} e_{2}\right)=x_{0}+x_{\hat{\imath}} \hat{\imath}$ can play a decisive role in the power series representation of any monogenic function. Of course, this is also true in the general case for $\mathbb{R}^{n+1}$ where one has to work analogously with a parameter set $\left(i_{1}, i_{2}, \ldots, i_{n}\right)$.

\section{Acknowledgements}

This work was supported by FEDER founds through COMPETE-Operational Programme Factors of Competitiveness ("Programa Operacional Factores de Competitividade") and by Portuguese funds through the Center for Research and Development in Mathematics and Applications (University of Aveiro) and the Portuguese Foundation for Science and Technology ("FCT-Fundação para a Ciência e a Tecnologia"), within project PEst-C/MAT/UI4106/2011 with COMPETE number FCOMP-01-0124-FEDER-022690. The research of the first author was also supported by FCT under the fellowship SFRH/BD/44999/2008.

\section{References}

1. Appell, P.: Sur une classe de polynômes. Ann. Sci. École Norm. Sup. 9(2), 119-144 (1880)

2. Bock, S., Gürlebeck, K.: On a generalized Appell system and monogenic power series. Math. Methods Appl. Sci. 33(4), 394-411 (2010)

3. Brackx, F., Delanghe, R., Sommen, F.: Clifford analysis. Pitman, Boston-LondonMelbourne (1982) 
4. Cruz, C., Falcão, M.I., Malonek, H.R.: On pseudo-complex bases for monogenic polynomials. In: Sivasundaram, S. (ed.) 9th International Conference on Mathematical Problems in Engineering, Aerospace and Sciences (ICNPAA 2012). AIP Conference Proceedings, vol. 1493, pp. 350-355 (2012)

5. Delanghe, R.: On regular-analytic functions with values in a Clifford algebra. Math. Ann. 185, 91-111 (1970)

6. Falcão, M.I., Cruz, J., Malonek, H.R.: Remarks on the generation of monogenic functions. 17th Inter. Conf. on the Appl. of Computer Science and Mathematics on Architecture and Civil Engineering, Weimar (2006)

7. Gürlebeck, K.: Über interpolation und approximation verallgemeinert analytischer funktionen. Wiss. Inf. 34, 21 S. (1982)

8. Gürlebeck, K., Malonek, H.: A hypercomplex derivative of monogenic functions in $\mathbb{R}^{n+1}$ and its applications. Complex Variables Theory Appl. 39, 199-228 (1999)

9. Gürlebeck, K., Sprößig, W.: Quaternionic analysis and elliptic boundary value problems, International Series of Numerical Mathematics, vol. 89. Birkhäuser Verlag, Basel (1990)

10. Gürlebeck, K., Habetha, K., Sprößig, W.: Holomorphic functions in the plane and $n$-dimensional space. Birkhäuser Verlag, Basel (2008)

11. Habetha, K.: Function theory in algebras. In: Complex analysis. Methods, trends, and applications, Math. Lehrb, vol. 61, pp. 225-237. Akademie-Verlag, Berlin (1983)

12. Malonek, H.: A new hypercomplex structure of the euclidean space $\mathbb{R}^{m+1}$ and the concept of hypercomplex differentiability. Complex Variables 14, 25-33 (1990)

13. Malonek, H.: Selected topics in hypercomplex function theory. In: Eriksson, S.L. (ed.) Clifford algebras and potential theory, pp. 111-150. 7, University of Joensuu (2004)

14. Malonek, H.R., Falcão, M.I.: On paravector valued homogeneous monogenic polynomials with binomial expansion. Advances in Applied Clifford Algebras 22 (3), 789-801 (2012) 